\title{
Occurrence of Fumonisins in Some Maize Meal Marketed to Consumers in Harare, Zimbabwe
}

\section{Princess Mushonga $^{1}$ \\ Wilson Parawira ${ }^{\star \star}$ \\ Loveness Kuziwa Nyanga ${ }^{3}$}

\author{
Department of Biological Sciences, Faculty of Science and Engineering, Bindura University of Science \\ Education, Chimurenga Road, Bindura, Zimbabwe. \\ ¿Email:parawiradr@yahoo.co.ukTel: +263716800026 \\ ${ }^{s}$ Institute of Food, Nutrition and Family Science, University of Zimbabwe, Mount Pleasant, Harare, Zimbabwe.
}

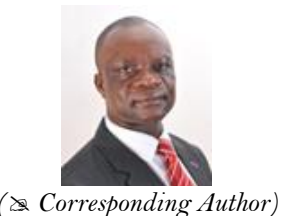

( Corresponding Author)

\begin{abstract}
Mycotoxins are toxic secondary metabolites secreted by filamentous fungi which affect animals, plants and humanscausing a lot of diseases. Fumonisins are the types that mostly affect cereal grains. The prevalence of fumonisins in Harare marketed maize meal was investigated in 72 randomly selected samples. Fumonisin B1 and Fumonisin B2 were extracted from maize meal with methanol-water $(3: 1, \mathrm{v} / \mathrm{v})$ using ultrasonic extraction. They were injected into an LC-MS system following centrifugation. A survey was also conducted to determine the measures taken by 5 major Harare millers to mitigate fumonisin contamination in the maize meal. Fumonisin B1 (FB1) was detected in all analysed samples at mean concentrations ranging between 61.45 and $265.79 \mu \mathrm{g} / \mathrm{kg}$. Fumonisin B2 (FB2) was detected in only $56.9 \%$ of the samples analysed, with a range between $13.72 \mu \mathrm{g} / \mathrm{kg}$ and $76.93 \mu \mathrm{g} / \mathrm{kg}$. The highest total fumonisin mean concentration $(\mathrm{FB} 1+\mathrm{FB} 2)$ was detected in maize meal with maize bran added to it $(342.72 \mu \mathrm{g} / \mathrm{kg})$, while the least total fumonisin mean concentration was detected in maize meal with wheat bran added to it $(61.45 \mu \mathrm{g} / \mathrm{kg})$. All the samples analysed were within the maximum tolerable limit (MTL) of 1000 $\mu \mathrm{g} / \mathrm{kg}$. However, the average probable daily intake (APDI) of fumonisins ranged between 0.82 and $4.57 \mu \mathrm{g} / \mathrm{kg}$ body weight/day. Maize meal with maize bran added and Roller meal had PDMI above the regulatory limit of $2 \mu \mathrm{g} / \mathrm{kg}$ body weight/day recommended by the World Health Organisation. The high frequency of fumonisins consumed daily in these two types of maize meal could be posing some health risks to consumers.
\end{abstract}

Keywords: Mycotoxins, Maize meal, Fumonisins, Milling, Risk assessment, World Health Organisation, Zimbabwe.

Citation | Princess Mushonga; Wilson Parawira; Loveness Kuziwa Nyanga (2020). Occurrence of Fumonisins in Some Maize Meal Marketed to Consumers in Harare, Zimbabwe. Agriculture and Food Sciences Research, 7(1): 58-64.

History:

Received: 22 January 2020

Revised: 28 February 2020

Accepted: 31 March 2020

Published: 5 May 2090

Licensed: This work is licensed under a Creative Commons Attribution 3.0 License (cc)

Publisher: Asian Online Journal Publishing Group
Acknowledgement: The authors would like to acknowledge the support they got from Bindura University of Science Education and the Pharmacy Department of the University of Zimbabwe for all the assistance throughout Department of the University of Zimbabwe for all the assistance throughout
our wuthors also want to thank all the interviewed Milling Companies for the information they provided for the successful completion of their work. Authors are indebted to Mr. C. Mutsimhu, Dr. B. Masamha and Mr. K. Basira who assisted them with the Statistical analyses.

Funding: This study received no specific financial support.

Competing Interests: The authors declare that they have no conflict of interests.

Transparency The authors confirm that the manuscript is an ho Transparency: The authors confirm that the manuscript is an honest,
accurate, and transparent account of the study was reported; that no vital features of the study have been omitted; and that any discrepancies from the study as planned have been explained.

Ethical: This study follows all ethical practices during writing.

\section{Contents}

1. Introduction

2. Materials and Methods

(a) 59

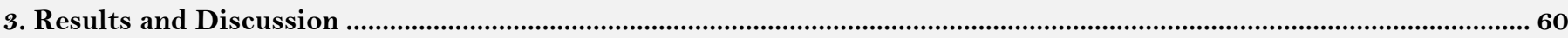

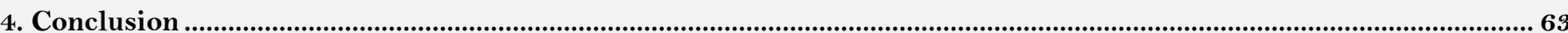

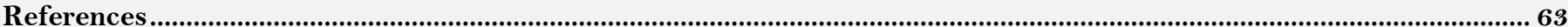




\section{Contribution of this paper to the literature}

The levels of fumonisins in all the maize meal analyzed in this study were below the maximum tolerable limits of $1000 \mu \mathrm{g} / \mathrm{kg}$ set by the European Union. However, the APDI of fumonisins estimated for maize meal with maize bran added and roller meal were above the provisional APDI set by WHO. Maize meal with maize bran added and Roller meal showed high contamination levels probably because they contained the bran and some germ that are highly contaminated by fumonisins. The high frequency of fumonisins consumed daily in both types of maize meal pose some health risks to consumers. There is need for setting up measures to check the quality of maize-based foods in order to alleviate the dangers of developing esophageal cancer and other probable health complications that are a result of fumonisin contamination.

\section{Introduction}

Maize (Zea mays L) is an economically significant cereal that is grown in Zimbabwe. It serves as the staple diet for the majority of the Zimbabwean populace. Maize is nutritionally important since it is an excellent source of proteins, starch, lipids, some bioactive compounds and some phytochemicals [1]. Maize may contain a number of important B vitamins, folic acid, Vitamin C, and provitamin A (i.e., precursor to vitamin A). Maize is also rich in phosphorus, magnesium, manganese, zinc, copper, iron and selenium, and has small amounts of potassium and calcium. It can be consumed as raw grain or processed into maize meal. However, safety can be greatly compromised when the maize grains are contaminated by fumonisins, the secondary organic metabolites secreted by Fusarium species [2].

Fumonisin $\mathrm{B}_{1}, \mathrm{~B}_{2}$ and $\mathrm{B}_{3}$ are the most predominant of all fumonisins and are often detected in maize and maize products [3]. A considerable volume of research on mycotoxins has been carried out in Zimbabwe on maize and studies have shown that fumonisin $\mathrm{B}_{1}$ mainly contaminates the maize grains before and after harvest [3, 4]. Fumonisin $B_{1}$ which accounts for nearly $70 \%$ of food contamination worldwide [5] is the most pernicious of the fumonisins. It has been associated with hepatocellular and oesophageal cancers, neural tube defects and impaired growth in children [6]. The B-series of fumonisins are generally thermo-stable at temperatures below $150{ }^{\circ} \mathrm{C}$ but their concentrations get significantly lower at temperatures above this value [2].

Insects, such as the maize stalk borer (Busseola fusca), infest maize cobs in the field and subsequently cause contamination of the grains by spreading mycotoxigenic fungi [7]. When water activity is high $\left(\mathrm{a}_{\mathrm{w}}>0.9\right)$, fumonisins are synthesised in maize before harvesting [8]. It is of paramount importance that maize kernels are adequately dried prior to milling in order to minimise the chances of contamination by the fumonisin-producingfungi.

Milling is a process that transforms grains into smaller consumable particles, such as maize meal, grits or starch. The milling process does not detoxify fumonisins, instead it re-distributes them, giving rise to higher or lower concentrations in the various milled fractions [9]. Studies have shown that the germ and the hull of the maize kernels harbour very high levels of fumonisins compared to the endosperm [10, 11$]$.

Zimbabwean milling companies buy maize from the Grain Marketing Board. They mill and package the maize meal which is then sold in the supermarkets. Recently, there has been an improvement in the nutritional composition of maize meal produced by some companies. Some are adding wheat bran or maize bran to the processed maize meal so as to enhance the fibre levels in their products. However, the brans might bring with them mycotoxins which contaminate the maize meal and pose some health risks to the consumers [12].

Fumonisins have been found to be the most prevalent mycotoxin in Zimbabwe. However, not much research has been done to determine levels of fumonisins in maize meal. The high frequencies of fumonisins observed in Zimbabwean maize grain are a cause for concern to human health [4]. Therefore, it is most appropriate to quantify the fumonisin levels in maize-meal since it is a direct product of maize consumed by many Zimbabweans. The findings will help to raise awareness on the levels and dangers of fumonisins in the different types of maize meal being consumed in the country.

\section{Materials and Methods \\ 2.1. Study Area}

The study was conducted in Harare, Zimbabwe where maize meal was bought from three different supermarkets. These three supermarkets represented the major supermarkets selling maize meal to the population (2 123132 people) in Harare (ZimStat. Census 2012: Provincial report). The supermarkets were selling maize meal from the Zimbabwean millers. Further, a survey was carried out on five major Zimbabwean millers to establish the mitigation measures during the milling process to reduce fumonisin contamination. Analysis of the maize meal samples was done at the laboratory of the Pharmacy Department of the University of Zimbabwe, Harare.

\subsection{Experimental Design}

This study used a descriptive approach. Quantitative observations were done by analysing and recording of quantifiable levels of fumonisin $\mathrm{B}_{1}$ and fumonisin $\mathrm{B}_{2}$ in the maize meal which was randomly purchased from Harare supermarket between September and December, 2018 from 5 different grinders/millers. Analysis of variance (ANOVA) was used to statistically analyse the results. Qualitative observation was done on the survey research. Both the quality controllers and those who were milling (millers) answered to questionnaires with both open-ended and close-ended questions which mainly focussed on the measures taken by these 5 millers to mitigate the effects of fumonisin and other mycotoxins contamination in maize meal.

\subsection{Sampling of Maize Meal}

From each of the five major Zimbabwean millers (M1, M2, M3, M4, M5), $2 \mathrm{~kg}$ maize-meal packets, 3 of each type (roller meal and super refined), were collected randomly from each of the three selected major supermarkets. In addition, $2 \mathrm{~kg}$ packets of each of super refined maize meal with wheat bran or maize bran were also collected from miller 2 using the same procedure as above. 
One hundred grams of each type of maize meal were drawn from each of the $2 \mathrm{~kg}$ packets at 3 points (bottom, middle and top of the pack). Similar types of maize meal samples collected from each individual miller were thoroughly mixed together at the laboratory of the Pharmacy Department, University of Zimbabwe. The mixed samples were sealed in sterile plastic bags and stored at $20^{\circ} \mathrm{C}$ until further analysis.

\subsection{Fumonisin Extraction from Maize Meal Samples}

Fumonisin extraction was done according to the protocol by Li, et al. [13] with Ultrasonic extraction which was modified. Maize meal samples were blended and $5 \mathrm{~g}$ of homogenized maize meal were mixed with $25 \mathrm{ml}$ of Extraction Solvent (methanol/water) in the ratio 3:1 (v/v). Triphenyl phosphate (TPP) was used as the internal standard. Samples were shaken by hand for 5 minutes and centrifuged at $4000 \mathrm{rpm}$ for 5 minutes using a Hettich Zentrifugen (Germany) centrifuge. The supernatant was placed into a $5 \mathrm{ml}$ syringe. It was then filtered, through a $0.22 \mathrm{~mm}$ acrodisc syringe filter, into an HPLC vial for LC-MS analysis.

\subsection{Calibration of Standards}

Five grams $(5 \mathrm{~g})$ of Blank (Probrand) Corn (corn without any fumonisin) were transferred into a $50 \mathrm{ml}$ falcon tube. The calibration standards and Quality Control Samples were matrix-matched by spiking known volumes of standards of $\mathrm{FB}_{1}$ and $\mathrm{FB}_{2}$ into blank samples to target concentrations $5 \mathrm{ng} / \mathrm{ml}, 10 \mathrm{ng} / \mathrm{ml}, 50 \mathrm{ng} / \mathrm{ml}, 100 \mathrm{ng} / \mathrm{ml}$, $500 \mathrm{ng} / \mathrm{ml}, 1000 \mathrm{ng} / \mathrm{ml}, 2000 \mathrm{ng} / \mathrm{ml}, 3000 \mathrm{ng} / \mathrm{ml}$ and $5000 \mathrm{ng} / \mathrm{ml}$. This was done using $25 \mathrm{ml}$ (methanol/water) in the ratio $3: 1(\mathrm{v} / \mathrm{v})$ as the diluent in which Triphenylphosphate (TPP) was used as the internal standard at 500 $\mathrm{ng} / \mathrm{ml}$. The tubes containing the sample and extraction solvent were capped and shaken vigorously for 5 minutes. They were further centrifuged for 5 minutes at $4000 \mathrm{rpm}$. The supernatant was put into a $5 \mathrm{ml}$ syringe and then filtered through $0.22 \mathrm{~mm}$ syringe filters into the vials for HPLC-MS analysis.

A calibration curve was made using 9 standards starting with $1000 \mathrm{ng} / \mathrm{ml}$ as stock. Spiked samples were produced to validate the method using QC standards: HQC-4000 $\mu \mathrm{g} / \mathrm{kg}$; MQC-2000 $\mu \mathrm{g} / \mathrm{kg}$; LQC- $50 \mu \mathrm{g} / \mathrm{kg}$.

\subsection{HPLC-MS Conditions for Fumonisin Analysis}

The HPLC used was Agilent HPLC 1260 System (California, USA) equiped with a binary pump, autosampler and thermostated column compartment. A Phenomenex, Luna Column with dimmesions 50 x $2 \mathrm{~mm}$, 3 micron was used for separation of the compounds. Mobile Phase A comprised $5 \mathrm{mM}$ Ammonium acetate; $\mathrm{pH}$ was adjusted with $16 \mathrm{ml}$ formic acid to 3.1. Mobile Phase B consisted of $100 \%$ methanol. The temperature of the column was maintained at $40{ }^{\circ} \mathrm{C}$. The flow rate was $0.45 \mathrm{ml} /$ minute while the injection volume was $80 \mu$ l. Fifty percent methanol was used for needle wash. Run time was set at $10 \mathrm{~min}$. All the fumonisins eluted at $7.2-7.8 \mathrm{~min}$.

Agilent Q-TOF 6530 Mass spectrometer was used for detection of fumonisins. It was fitted with an electrospray ionisation (ESI) probe and operated in the positive ionisation mode. The following parameters were optimised: capillary voltage, $4000 \mathrm{~V}$; drying gas temperature, $350{ }^{\circ} \mathrm{C}$ and desolvation gas flow rate, $10 \mathrm{l} / \mathrm{min}$. Data acquisition and analysis was done using the Mass Hunter software version B.07.03 (509).

\subsection{Method Validation}

An 'in house' validation was applied according to the internal procedure as well as the acceptance criteria for Bio-analytical Method Development and Validation. Intra-day accuracy (97.54 \%), linearity (0.995-0.999) and precision (2.78\%) of the quality control (Q C) samples for Fumonisin $\mathrm{B}_{1}$ and Fumonisin $\mathrm{B}_{2}$ were within the accepted range. Inter-day quality control samples for both Fumonisin $\mathrm{B}_{1}$ and Fumonisin $\mathrm{B}_{2}$ also passed the acceptance criteria. The average recoveries for all quality control samples were between $85 \%$ and $96 \%$. Percent Coefficient of Variation was less than $2.78 \%$ for all QC samples. Calibration curves for both Fumonisin $\mathrm{B}_{1}$ and Fumonisin $\mathrm{B}_{2}$ showed that linearity were greater than 0.9900 according to the set regulations for method development and validation. Limit of detection for Fumonisin $B_{1}$ and Fumonisin $B_{2}$ were $5 \mu \mathrm{g} / \mathrm{kg}$. In this regard, the LC-MS method developed passed all the acceptance criteria in accordance with FDA principles for method validation and related regulations; therefore it was fit for routine sample analysis for fumonisins in maize meal.

\subsection{Estimation of the Probable Average Daily Intake of Fumonisins in the 4 Types of Maize Meal}

The estimation of the probable average daily intake (APDI) was done by calculating the average probable daily intake of $\mathrm{FB}_{1}$ and $\mathrm{FB}_{2}$ combined in the samples. The estimates of the average probable daily intake (APDI) of $\mathrm{FB}{ }_{1}$ and $\mathrm{FB}_{2}$ combined in the samples were calculated using the formula:

$\mathrm{APDI}=\mathrm{L} \times \mathrm{D} / 60 \mathrm{~kg}$, where:

APDI is the average probable daily intake. $\mathrm{L}$ is the mean total concentration of $\mathrm{FB}_{1}+\mathrm{FB}_{2}$ in the maize meal samples. $\mathrm{D}$ is daily consumption of maize meal - average of $800 \mathrm{~g}[3,4]$. The proposed typical body weight of an adult was $60 \mathrm{~kg}$.

\subsection{Data Analysis}

Quantitative data were analyzed using Unbalanced Analysis of variance (ANOVA) performed in Genstat Version 19. Mean differences in the levels of fumonisin contamination were determined using least significant differences (LSD) at $5 \%$ level of significance. An Unbalanced Design ANOVA was used because there was no equal number of observations since some fumonisin quantities were below the limit of detection. The qualitative data collected using the questionnaires were analyzed using content analysis.

\section{Results and Discussion}

\subsection{Survey Results}

A questionnaire was administered to the millers and quality controllers of each milling company to establish the mitigation measures these milling companies were taking to reduce the levels of fumonisins in their maize meal and the responses are summarized in Table 1. Most of them knew very little about fumonisins, instead, during their quality checks they focused on aflatoxins, which are less abundant in Zimbabwean maize than fumonisins [3, 4]. 
Their lack of knowledge about fumonisins may pre-dispose the maize meal consumers to health risks from fumonisin contamination. Millers were not monitoring fumonisin levels in both the maize and the maize meal. This has potentially detrimental effects on the consumer's health. Mwalwayo and Thole [14] stressed the cooccurrence of aflatoxins with fumonisins in maize; therefore millers need to use cheaper and effective methods of testing for multiple mycotoxins in the maize meal, such as the liquid chromatography mass spectrometry technique (HPLC/MS) [15]. All the millers were sorting and cleaning the maize kernels prior to milling. They did the sorting to remove physically damaged and infected grain based on physical damage and the presence of moulds. Cleaning of the maize was done to remove cobs, chuff and stones. The sorting could explain the lower mean concentrations of fumonisins obtained in this study. Research done by Pietri, et al. [11] showed that fumonisin concentrations were lower in the cleaned maize than the unprocessed kernels. Santini, et al. [16] also noted that cleaning, if done effectively, reduces fumonisins concentrations from the pericarp as well as from the damaged and broken grains by $50 \%$. Matumba, et al. [17] and Kimanya, et al. [18] also allude to the fact that sorting of maize prior to milling reduces contamination of maize by fumonisins since defective kernels are discarded.

Table-1. Miller's responses on mitigation measures to reduce fumonisin contamination in maize meal.

\begin{tabular}{|c|c|c|c|c|c|}
\hline Parameter & Miller 1 & Miller 2 & Miller 3 & Miller 4 & Miller 5 \\
\hline Vetting of grain for weevils & $\checkmark$ & $\sqrt{ }$ & $\checkmark$ & $\checkmark$ & $\sqrt{ }$ \\
\hline Monitoring moisture level & $\checkmark$ & $\checkmark$ & $\checkmark$ & $\checkmark$ & $\checkmark$ \\
\hline Treatment prior to storage with insecticides & $\mathrm{x}$ & $\checkmark$ & $\mathrm{x}$ & $\mathrm{x}$ & $\checkmark$ \\
\hline Covering of bags or silos & $\mathrm{x}$ & $\mathrm{X}$ & $\checkmark$ & $\checkmark$ & $\checkmark$ \\
\hline Cleaning of storehouse before storage & $\checkmark$ & $\checkmark$ & $\checkmark$ & $\checkmark$ & $\checkmark$ \\
\hline Removal of old grain before adding new grain & $\checkmark$ & $\checkmark$ & $\checkmark$ & $\checkmark$ & $\checkmark$ \\
\hline Use of pesticides during storage & $\mathrm{x}$ & $\checkmark$ & $\checkmark$ & $\checkmark$ & $\checkmark$ \\
\hline Sampling to monitor aflatoxin levels & $\checkmark$ & $\checkmark$ & $\checkmark$ & $\checkmark$ & $\checkmark$ \\
\hline Analysis for fumonisins before milling & $\mathrm{x}$ & $\mathrm{X}$ & $\mathrm{x}$ & $\mathrm{x}$ & $\mathrm{X}$ \\
\hline Sorting of grain & $\checkmark$ & $\checkmark$ & $\checkmark$ & $\checkmark$ & $\checkmark$ \\
\hline Testing for fumonisins in the maize meal & $\mathrm{x}$ & $\mathrm{X}$ & $\mathrm{x}$ & $\mathrm{x}$ & $\mathrm{X}$ \\
\hline
\end{tabular}

However, despite the cleaning and sorting processes, some fumonisins were detected. This could be attributed to the cleaning method (screening and sifting), which did not completely eliminate fumonisins but only served to reduce them. Nyangi [7] reported that the most noticeable sign of Fusarium infection in maize grown in the tropical areas is kernel rot. However, fumonisins are often detected in maize kernels even if in the absence of invisible signs of infection.

All the millers interviewed stressed that they checked the moisture content of their grain upon receipt from the Grain Marketing Board (GMB). This mitigation measure helped in the prevention of moulds that cause fumonisin contamination. Bacon and Nelson [19] state that levels of kernel contamination by fumonisins under improper storage and the optimal growth condition for fumonisin-producing moulds are when moisture content of harvested maize is between $18-23 \%$. The monitoring of moisture levels within this range by these millers could be another reason for the low fumonisin levels detected in the maize meal analyzed in this study.

Millers stored their grain in silos and this made them more susceptible to cross-contamination by fumonisins. The use of hermetic technology for grain storage could be an effective alternative method in the reduction of fumonisins since the bags or silos suffocate the insects and fungi, preventing contamination by fumonisins. Hove, et al. [4] noted that maize stored in polypropylene bags was less contaminated than un-bagged maize (mean $\mathrm{FB}_{1}=263 \mu \mathrm{g} / \mathrm{kg}$ and $401 \mu \mathrm{g} / \mathrm{kg}$ respectively). Most of the millers interviewed indicated that in the silos, tobacco weevil was the most challenging. Weevils feed upon the kernels and at the same time spread the infection to other healthy kernels. In spite of vetting the grain for weevils upon its reception from the GMB, the weevils could mature in the grains stored in the silos, leading to contamination. Miller 1 indicated that they did not apply any insecticides during storage of the grain. This could be the reason for the highest level of fumonisin contamination compared to other millers. Other 4 millers were applying Phosphin gas tablets at a rate of 10 tablets per silo so as to kill the weevils in order to reduce contamination by fumonisins. This data shows that fumonisin control was not intentional, rather indirect through direct practices destined to assure the control of food safety and quality.

\subsection{Fumonisin Levels in Maize Meal}

Analysis was done on the types of maize meal from different millers to determine fumonisin levels. There was a significant difference among the 5 millers in the concentration of fumonisins in the maize meal. There was also a significant difference between Fumonisin $B_{1}$ and Fumonisin $B_{2}$ levels in the 4 types of maize meal see Table 2. Again, a difference existed in the fumonisin concentration among the 4 types of maize meal (Roller meal, Super refined, Super refined with maize bran and Super refined with wheat bran).

From the 72 samples analysed from the marketed maize meal produced by the large millers, all maize meal samples (roller meal, super refined, maize meal with wheat bran and maize meal with maize bran) had a fumonisin $\mathrm{B}_{1}$ level below the maximum tolerable limit (MTL) of $1000 \mu \mathrm{g} / \mathrm{kg}[20]$ see Table 2. Of the samples analysed for $\mathrm{FB}_{2} 56.9 \%$ had non-detectable levels. Although half the samples of super refined maize meal had concentrations below the detection limit, all maize meal samples with wheat bran added did not present detectable concentrations of fumonisin $\mathrm{B}_{2}$. There was much variability in $\mathrm{FB}_{2}$ levels of super refined and this could be attributed to the low sample size.

Results from this study also showed mean fumonisin levels in the maize meal samples ranging from 61.64 $\mu \mathrm{g} / \mathrm{kg}$ to $265.79 \mu \mathrm{g} / \mathrm{kg}$ for $\mathrm{FB}_{1}$ and $0.00 \mu \mathrm{g} / \mathrm{kg}$ to $76.93 \mu \mathrm{g} / \mathrm{kg}$ for $\mathrm{FB}_{2}$ Table 2 . All analysed samples had $\mathrm{FB}_{1}$ but only $43.1 \%$ were contaminated by $\mathrm{FB}_{2}$. The lower concentrations of fumonisins observed, compared to other studies $[3,4,21]$ could reflect the effectiveness of the measures taken by millers to indirectly assure the control of safety and quality of foods. 
A research conducted by Doko, et al. [21] on Zimbabwean maize meal showed that $83 \%$ of the analysed samples had fumonisin $\mathrm{B}_{1}, \mathrm{~B}_{2}$ and $\mathrm{B}_{3}$ ranging between $55 \mu \mathrm{g} / \mathrm{kg}$ and $2735 \mu \mathrm{g} / \mathrm{kg}$. Murashiki, et al. [3] recorded fumonisin concentrations ranging between $10.43 \mu \mathrm{g} / \mathrm{kg}$ and $606.64 \mu \mathrm{g} / \mathrm{kg}$ in maize meal samples from Shamva and Makoni Districts $(P<0.05)$. These researchers analysed samples from small-holder farmers who took no precautionary measures against fumonisin contamination in the grains as large millers do, thus the high fumonisin levels detected.

Fumonisin $B_{1}$ and $B_{2}$ mean concentrations were significantly higher in maize meal with maize bran than other maize meal types $($ mean $=265.79 \mu \mathrm{g} / \mathrm{kg}$ ). Roller meal had fumonisin mean concentrations which were slightly lower than those found in the maize meal with maize bran but higher than in super refined maize meal Table 2 . This is because the bran is the most colonized by the Fusarium spp., followed by the germ fraction. These results are comparable to Pietri, et al. [11], Brera, et al. [22] and Vanara, et al. [23] who reported that when the corn is milled, fumonisins are in elevated concentrations in the bran, followed by the germ fraction. Broggi and others carried out a study in a commercial dry-mill in Argentina and found a three-fold increase in fumonisin contamination in the germ and bran fractions than in the whole corn [7].

Roller meal samples had fumonisin levels higher than super-refined maize meal samples because some pericarp and germ fragments probably found their way into the course meal during dry- milling [24]. Even though there was no bran or germ added to the super-refined maize meal, some fumonisins $(80.49 \mu \mathrm{g} / \mathrm{kg})$, were recovered from the samples. The main cause of contamination could be due to the floury endosperm that surrounds the germ where a higher formation of fumonisins is possible. In support of this, Vanara, et al. [2] noted that the presence of amylopectin in the endosperm during kernel development instigates $\mathrm{FB}_{1}$ biogenesis. A study by Gwirtz and Garcia-Casal [25] on processing of maize flour and corn meal products also showed a remarkable lowering of fumonisin concentrations in the endosperm during dry milling.

Levels of fumonisins obtained in maize meal where wheat bran was added to super refined were very low. At 5 $\%$ significance level, there was no difference between super -refined maize meal and super refined maize meal with wheat added Table 2 . This indicates that wheat was not a source of contamination by Fusarium compared to maize. Streit, et al. [26] state that fumonisin contamination commonly occurs in maize and maize products.

However, although the results show that fumonisin concentration in the types of maize meal are within the maximum tolerable limit (MTL) of $1000 \mu \mathrm{g} / \mathrm{kg}$ by EU standard $[12,14]$ there is need for constant monitoring of fumonisin levels, especially in the maize meal with maize bran added and in the roller meal.

Fumonisin $B_{1}$ was found to be more abundant in the maize meal samples than fumonisin $\mathrm{B}_{2}$ Table 2 . At a least significance difference of $5 \%$, the results from this research showed that there was a significant difference in the contamination of maize meal by the two types of fumonisins. Fumonisin $\mathrm{B}_{2}$ was not detected in maize meal samples, such as maize meal with wheat bran added and some super refined maize meal yet $\mathrm{FB}_{1}$ was detected in the same samples at $2.5 \mu \mathrm{g} / \mathrm{kg}$ detection limit. This also confirmed that Fumonisin $\mathrm{B}_{1}$ is the dominant type that contaminates Zimbabwean maize. This is in line with the observations by Marasas, et al. [27] and Rheeder, et al. [28] who noted that $\mathrm{FB}_{1}$ pre-dominates and contributes $70-80 \%$ of all fumonisins whereas $\mathrm{FB}_{2}$ constitutes $15-25$ $\%$ in maize grains. The differences in quantity between $\mathrm{FB}_{1}$ and $\mathrm{FB}_{2}$ could be because moulds produce higher quantities of the former.

Table-2. Means of fumonisin concentration $(\mu \mathrm{g} / \mathrm{kg})$ for the different products from all millers combined.

\begin{tabular}{c|c|c|c|c}
\hline Fumonisin type & $\begin{array}{c}\text { Maize meal with } \\
\text { maize bran }\end{array}$ & Roller meal & Super refined & $\begin{array}{c}\text { Maize meal with } \\
\text { wheat bran }\end{array}$ \\
\hline $\mathrm{FB}_{1}$ & $265.79 \pm 22.22^{\mathrm{a}}$ & $229.85 \pm 10.28^{\mathrm{b}}$ & $80.49 \pm 10.28^{\mathrm{c}}$ & $61.45 \pm 22.22^{\mathrm{c}}$ \\
\hline $\mathrm{FB}_{2}$ & $76.93 \pm 31.43^{\mathrm{c}}$ & $32.83 \pm 12.15^{\mathrm{d}}$ & $13.72 \pm 14.57^{\mathrm{d}}$ & $0.0^{\mathrm{e}}$ \\
\hline
\end{tabular}

the same at $5 \%$ significance level.
the

Analysis was done to assess fumonisin mean concentration in the types of maize meal produced by the different millers. Miller 1 had the highest mean fumonisin $B_{1}$ concentration of $336.40 \mu \mathrm{g} / \mathrm{kg}$ Table 3 . The high levels in samples from Miller 1, compared to other millers, may be attributed to the fact that they were not treating their maize grains prior to storage and as a result, weevils could have probably attacked the grains whilst in silos and caused further contamination by fumonisins. Again, they did not cover the silos so it was easier for the insects to spread around and cause further contamination in, otherwise healthy, grains. Hove, et al. [4] also noted that the damage caused by pests contributes a lot to mycotoxin contamination of the maize grains. That is why it is of paramount importance to apply chemicals that protect the grain from damage.

Miller 2 recorded the second highest mean levels of Fumonisin $B_{1}(158.39 \mu \mathrm{g} / \mathrm{kg})$ because it was the only interviewed Milling Company that manufactures maize meal with maize bran added to it. As has been mentioned earlier on, the bran fraction contains the highest fumonisin levels in maize grains. Furthermore, Miller 2 was not covering the silos so this may have caused cross- contamination of grain by weevils. At $5 \%$ level of significance, there was no outstanding difference in fumonisin $B_{1}$ mean concentration among millers 3 , 4 and 5 . They all recorded the low mean concentration. Miller 2 showed a marked difference from all other miller as it contained high levels of fumonisin $\mathrm{B}_{2}(81.87 \mu \mathrm{g} / \mathrm{kg})$. This could also be attributed to the fact that they produced maize meal with maize bran, which normally contains a significant amount of fumonisins.

Table-3. Overall fumonisin mean concentration $(\mu \mathrm{g} / \mathrm{kg})$ for different millers for all types of maize meal combined.

\begin{tabular}{c|c|c|c|c|c}
\hline Fumonisin type & Miller 1 & Miller 2 & Miller 3 & Miller 4 & Miller 5 \\
\hline $\mathrm{FB}_{1}$ & $336.40 \pm 15.7^{\mathrm{a}}$ & $158.39 \pm 13.8^{\mathrm{b}}$ & $75.62 \pm 15.7^{\mathrm{c}}$ & $94.96 \pm 15.8^{\mathrm{c}}$ & $122.40 \pm 15.7^{\mathrm{c}}$ \\
\hline $\mathrm{FB}_{2}$ & $10.05 \pm 18.6^{\mathrm{d}}$ & $81.87 \pm 19.5^{\mathrm{ce}}$ & $3.1419 .1^{\mathrm{d}}$ & $25.34 \pm 31.4^{\mathrm{d}}$ & $19.60 \pm 19.1^{\mathrm{d}}$ \\
\hline
\end{tabular}

the same at $5 \%$ significance level. 


\subsection{Estimate of the Average Probable Daily Intake (APDI) for Risk Assessment}

The magnitude of fumonisin contamination obtained in the present study was below the tolerable regulatory level of $1000 \mu \mathrm{g} / \mathrm{kg}$ which was set by the European Commission [20]. However, the Joint FAO/WHO expert committee on food additives (JECFA) set the health-based guidance value for $\mathrm{FB}_{1}, \mathrm{FB}_{2}$ and $\mathrm{FB}_{3}$ separately or combined at $2 \mu \mathrm{g} / \mathrm{kg}$ body weight/day [29]. In this study, risk assessment was done on the four types of maize meal to establish levels to which maize meal consumers are exposed to fumonisins daily.

This study showed the Average Probable Daily Intake (APDI) of fumonisins by consumers Table 4. The values for maize meal with maize bran added and roller meal were above the set value of $2 \mu \mathrm{g} / \mathrm{kg}$ body weight/day. It is an indication that consumers of these types of maize meal with maize bran in Harare, Zimbabwe, are at greater risk of vulnerability due to the detrimental accumulative levels of fumonisins unlike consumers of super refined maize meal and maize meal with wheat bran added. Moreover, low level chronic intake of fumonisins in the maize and maize products can be more devastating than one-time high level intake A study carried out by Williams, et al. [30] reported that 4.5 billion maize consumers in the developing countries are exposed to chronic toxicity.

The study conducted by Murashiki, et al. [3] showed that populations in Shamva and Makoni Districts, Zimbabwe, had a Probable Daily Intake of 5.76-8.09 $\mu \mathrm{g} / \mathrm{kg}$ body weight/day. These figures are slightly higher than the values calculated in this study, most probably due to the mitigation measures taken by the registered millers and the type of milling done to reduce fumonisin contamination in the maize meal unlike with unregistered millers used by Murashiki, et al. [3]. Further, analysis done by Hove, et al. [4] showed a Maximum Probable Daily Intake of $5.40 \mu \mathrm{g} / \mathrm{kg}$ body weight/day for children between $5-9$ years, $4.40 \mu \mathrm{g} / \mathrm{kg}$ body weight/day for adolescents and $2.30 \mu \mathrm{g} / \mathrm{kg}$ body weight/day for adults in Zimbabwe. High fumonisin intakes $(141.97 \mu \mathrm{g} / \mathrm{kg}$ body weight/day) have also been reported in Tanzania by Kimanya, et al. [18].

Other studies in the more economically developed countries, such as Spain and Brazil, have indicated fumonisin levels as low as $0.1 \mu \mathrm{g} / \mathrm{kg}$ body weight/day and $0.063 \mu \mathrm{g} / \mathrm{kg}$ body weight/day respectively [31]. These low values are perhaps due to the advanced and effective mitigation measure taken by these countries to mitigate fumonisin contamination in foods. This is coupled to the low intake of maize-based foods [31] unlike in Zimbabwe where the staple diet is primarily maize-based. On grounds of the high Average Probable Daily Intake of fumonisins detected in some types of maize meal being sold in Harare markets, it is recommended to adopt some measures to mitigate the deleterious effects of fumonisin consumption. These mitigation practices include; sorting and cleaning of grain to remove broken and moldy kernels, application of grain-protection chemicals to kill weevils and monitoring of moisture levels to reduce contamination of grain by fumonisins [29].

\begin{tabular}{l|c|c} 
Table-4. Total mean fumonisin concentrations $\left(\mathrm{FB}_{1}+\mathrm{FB}_{2}\right)$ and probable intakes of marketed maize meal in Harare, Zimbabwe. \\
\hline Type of maize meal & $\begin{array}{c}\text { Total mean fumonisin } \\
\text { concentration }(\boldsymbol{\mu g} / \mathbf{k g})\end{array}$ & $\begin{array}{c}\text { Average Probable Daily Intake } \\
(\boldsymbol{\mu g} / \mathbf{k g} \mathbf{~ b w} / \mathbf{d a y})\end{array}$ \\
\hline Maize meal with maize bran added & 342.72 & 4.57 \\
\hline Roller meal & 262.68 & 3.50 \\
\hline Super refined & 94.21 & 1.26 \\
\hline Maize meal with wheat bran added & 61.45 & 0.82 \\
\hline
\end{tabular}

\section{Conclusion}

The levels of fumonisins in all the maize meal analyzed in this study were below the maximum tolerable limits of $1000 \mu \mathrm{g} / \mathrm{kg}$ set by the European Union. However, the APDI of fumonisins estimated for maize meal with maize bran added and roller meal were above the provisional APDI set by WHO. Maize meal with maize bran added and Roller meal showed high contamination levels probably because they contained the bran and some germ that are highly contaminated by fumonisins. The high frequency of fumonisins consumed daily in both types of maize meal pose some health risks to consumers. There is need for setting up measures to check the quality of maize-based foods in order to alleviate the dangers of developing esophageal cancer and other probable health complications that are a result of fumonisin contamination.

Results from this study also suggested that some of the practices that are being taken by the millers are effective at reducing fumonisin contamination in the maize meal. The milling process itself, which includes dehulling and de-germination of maize kernels, seems to dilute and re- distribute fumonisins into fractions that mostly become animal feed. It is of paramount importance to undertake further research on small- scale millers that will assist them to meet international quality standards and adopt those practices that minimize risks to fumonisin contamination in the maize meal they produce.

\section{References}

[1] S. Siyuan, L. Tong, and H. L. Rui, "Corn phytochemicals and their health benefits," Journal of Food Science and Human Wellness, vol. 3, pp. 185-195, 2018.

[2] F. Vanara, V. Scarpino, and M. Blandino, "Fumonisin distribution in maize dry-milling products and by-products: Impact of two industrial degermination systems," Toxins, vol. 10, p. 357, 2018. Available at: https://doi.org/10.3390/toxins 10090357.

[3] T. C. Murashiki, C. Chidewe, M. A. Benhura, D. T. Maringe, M. P. Dembedza, L. R. Manema, B. M. Mvumi, and L. K. Nyanga, "Levels and daily intake estimates of aflatoxin B1 and fumonisin B1 in maize consumed by rural households in Shamva and Makoni districts of Zimbabwe," Food Control, vol. 72, pp. 105-109, 2017. Available at: https://doi.org/10.1016/j.foodcont.2016.07.040.

[4] M. Hove, M. De Boevre, C. Lachat, L. Jacxsens, L. Nyanga, and S. De Saeger, "Occurrence and risk assessment of mycotoxins in subsistence farmed maize from Zimbabwe," Food Control, vol. 69, pp. 36-44, 2016. Available at: https://doi.org/10.1016/j.foodcont.2016.04.038.

[5] P. G. Thiel, W. F. Marasas, E. W. Sydenham, G. S. Shephard, and W. C. Gelderblom, "The implications of naturally occurring levels of fumonisins in corn for human and animal health," Mycopathologia, vol. 117, pp. 3-9, 1992. Available at: https://doi.org/10.1007/bfo0497272.

[6] M. E. Zain, "Impact of mycotoxins on humans and animals," Journal of Saudi Chemical Society, vol. 15, pp. 129-144, 2011. Available at: https://doi.org/10.1016/j.jscs.2010.06.006.

[7] C. Nyangi, "Aflatoxin and fumonisin contamination of maize and beans along the food and feed value chain in Babati District, Tanzania," MSc Thesis. Sokoine University of Agriculture, Morogoro, Tanzania, 2014.

[8] S. Marín Sillué, A. J. Ramos Girona, G. Cano Sancho, and V. Sanchís Almenar, "Mycotoxins: Occurrence, toxicology, and exposure assessment," Food and Chemical Toxicology, vol. 60, pp. 218-237, 2013. Available at: https://doi.org/10.1016/j.fct.2013.07.047. 
[9] L. B. Bullerman and A. Bianchini, "Stability of mycotoxins during food processing," International Journal of Food Microbiology, vol. 119, pp. 140-146, 2007. Available at: https://doi.org/10.1016/j.ijfoodmicro.2007.07.035.

[10] International Agency for Research on Cancer (IARC), Some traditional herbal medicines, some mycotoxins, naphthalene, and styrene. Monographs on the evaluation of the carcinogenic risk of chemicals to humans vol. 82. Lyon, France: IARC Press, 2002.

[11] A. Pietri, M. Zanetti, and T. Bertuzzi, "Distribution of aflatoxins and fumonisins in dry-milled maize fractions," Food Additives \& Contaminants: Part A, vol. 26, pp. 372-380, 2009. Available at: https://doi.org/10.1080/02652030802441513.

[12] N. Nleya, M. C. Adetunji, and M. Mwanza, "Current status of mycotoxin contamination of food commodities in Zimbabwe," Toxins, vol. 10, p. 89, 2018. Available at: https://doi.org/10.3390/toxins 10050089 .

[13] C. Li, Y.-L. Wu, T. Yang, and W.-G. Huang-Fu, "Rapid determination of fumonisins B1 and B2 in corn by liquid chromatographytandem mass spectrometry with ultrasonic extraction," Journal of Chromatographic Science, vol. 50, pp. 57-63, 2012. Available at: https://doi.org/10.1093/chromsci/bmroo9.

[14] D. Mwalwayo and B. Thole, "Prevalence of aflatoxin and fumonisins (B1+ B2) in maize consumed in rural Malawi," Toxicology Reports, vol. 3, pp. 173-179, 2016. Available at: https://doi.org/10.1016/j.toxrep.2016.01.010.

[15] P. Martos, W. Thompson, and G. Diaz, "Multiresidue mycotoxin analysis in wheat, barley, oats, rye and maize grain by highperformance liquid chromatography-tandem mass spectrometry," World Mycotoxin Journal, vol. 3, pp. 205-223, 2010. Available at: https://doi.org/10.3920/wmj2010.1212.

[16] A. Santini, A. Raiola, G. Meca, and A. Ritieni, "Aflatoxins, ochratoxins, trichotecenes, patulin, fumonisins and beauvericin in finished products for human consumption," Journal of Clinical Toxicology, vol. 5, pp. 2-11, 2015. Available at: https://doi.org/10.4172/2161-0495.1000265.

[17] L. Matumba, C. Van Poucke, E. Njumbe Ediage, B. Jacobs, and S. De Saeger, "Effectiveness of hand sorting, flotation/washing, dehulling and combinations thereof on the decontamination of mycotoxin-contaminated white maize," Food Additives \&ٔ Contaminants: Part A, vol. 32, pp. 960-969, 2015. Available at: https://doi.org/10.1080/19440049.2015.1029535.

[18] M. E. Kimanya, B. De Meulenaer, B. Tiisekwa, M. Ndomondo-Sigonda, F. Devlieghere, J. Van Camp, and P. Kolsteren, "Cooccurrence of fumonisins with aflatoxins in home-stored maize for human consumption in rural villages of Tanzania," Food additives and contaminants, vol. 25, pp. 1353-1364, 2008

[19] C. W. Bacon and P. E. Nelson, "Fumonisin production in corn by toxigenic strains of Fusarium moniliforme and Fusarium proliferatum," Journal of Food Protection, vol. 57, pp. 514-52 1, 1994. Available at: https://doi.org/10.4315/0362-028x-57.6.514

[20] European Commission, "Commission regulation (EC) No 1126/2007 of 26 September 2007 amending regulation (EC) No 1881/2006: Setting maximum levels for certain contaminants in foodstuffs as regards Fusarium toxins in maize and maize products," Journal of European Union Legislation, vol. 254, pp. 14-17, 2007.

[21] M. B. Doko, C. Canet, N. Brown, E. W. Sydenham, S. Mpuchane, and B. A. Siame, "Natural co-occurrence of fumonisins and zearalenone in cereals and cereal-based foods from Eastern and Southern Africa," Journal of Agricultural and Food Chemistry, vol. 44, pp. 3240-3243, 1996. Available at: https://doi.org/10.1021/jf960257+.

[22] C. Brera, F. Debegnach, S. Grossi, and M. Miraglia, "Effect of industrial processing on the distribution of fumonisin B1 in dry milling corn fractions," Journal of Food Protection, vol. 67, pp. 1261-1266, 2004. Available at: https://doi.org/10.4315/0362-028x67.6.1261.

[23] F. Vanara, A. Reyneri, and M. Blandino, "Fate of fumonisin B1 in the processing of whole maize kernels during dry-milling," Food Control, vol. 20, pp. 235-238, 2009. Available at: https://doi.org/10.1016/j.foodcont.2008.05.014.

[24] M. Castells, S. Marín, V. Sanchis, and A. J. Ramos, "Distribution of fumonisins and aflatoxins in corn fractions during industrial cornflake processing," International Journal of Food Microbiology, vol. 123, pp. 81-87, 2008. Available at: https://doi.org/10.1016/j.ijfoodmicro.2007.12.001.

[25] J. A. Gwirtz and M. N. Garcia-Casal, "Processing maize flour and corn meal food products," Annals of the New York Academy of Sciences, vol. 1312, pp. 66-75, 2014. Available at: https://doi.org/10.1111/nyas.12299.

[26] E. Streit, G. Schatzmayr, P. Tassis, E. Tzika, D. Marin, I. Taranu, C. Tabuc, A. Nicolau, I. Aprodu, and O. Puel, "Current situation of mycotoxin contamination and co-occurrence in animal feed-Focus on Europe," Toxins, vol. 4, pp. 788-809, 2012. Available at: https://doi.org/10.3390/toxins4100788.

[27] W. Marasas, W. Gelderblom, G. S. Shephard, and H. Vismer, Mycotoxins: A global problem. In: Mycotoxins: Detection methods, management, public health and agricultural trade. (Edited by Leslie, J.F. Bandyopadhyay, R. and Visconti, A.). Townbridge, UK: Cromwell Press, 2008.

[28] J. P. Rheeder, W. F. Marasas, and H. F. Vismer, "Production of fumonisin analogs by Fusarium species," Journal of Applied Environmental Microbiology, vol. 68, pp. 2101-2 105, 2002. Available at: https://doi.org/10.1128/aem.68.5.2101-2105.2002.

[29] World Health Organization, "Food and safety digest. Department of food safety and zooness. Retrieved from: https://www.who.int/foodsafety/foodsafetydigest/en/," 2018.

[30] J. H. Williams, T. D. Phillips, P. E. Jolly, J. K. Stiles, C. M. Jolly, and D. Aggarwal, "Human aflatoxicosis in developing countries: A review of toxicology, exposure, potential health consequences, and interventions," The American Journal of Clinical Nutrition, vol. 80, pp. 1106-1h122, 2004. Available at: https://doi.org/10.1093/ajcn/80.5.1106.

[31] G. Cano-Sancho, A. J. Ramos, S. Marín, and V. Sanchis, "Occurrence of fumonisins in Catalonia (Spain) and an exposure assessment of specific population groups," Food Additives \& Contaminants: Part A, vol. 29, pp. 799-808, 2012. Available at: https://doi.org/10.1080/19440049.2011.644813. 